Documentation et bibliothèques

\title{
L’Association québécoise pour l'étude de l'imprimé : un lieu de réflexion et d'échanges autour du livre
}

\section{Jacques Michon et Gilles Gallichan}

Volume 37, numéro 1, janvier-mars 1991

URI : https://id.erudit.org/iderudit/1028410ar

DOI : https://doi.org/10.7202/1028410ar

Aller au sommaire du numéro

Éditeur(s)

Association pour l'avancement des sciences et des techniques de la

documentation (ASTED)

ISSN

0315-2340 (imprimé)

2291-8949 (numérique)

Découvrir la revue

Citer ce document

Michon, J. \& Gallichan, G. (1991). L'Association québécoise pour l'étude de

l'imprimé : un lieu de réflexion et d'échanges autour du livre. Documentation et

bibliothèques, 37(1), 37-38. https://doi.org/10.7202/1028410ar

Tous droits réservés $@$ Association pour l'avancement des sciences et des techniques de la documentation (ASTED), 1991
Ce document est protégé par la loi sur le droit d'auteur. L'utilisation des services d'Érudit (y compris la reproduction) est assujettie à sa politique d'utilisation que vous pouvez consulter en ligne.

https://apropos.erudit.org/fr/usagers/politique-dutilisation/ 


\section{L'Association québécoise pour l'étude de l'imprimé : un lieu de réflexion et d'échanges autour du livre}

Lorsque, dans l'avenir, les historiens se pencheront sur la place de l'imprimé en Occident à la fin du $X X^{e}$ siècle, ils ne manqueront pas de souligner l'impact des nouvelles technologies informatiques sur ce moyen de communication sociale. Comme l'arrivée de la presse à imprimer au $X V$ e siècle a révolutionné la civilisation européenne, l'informatique et la télématique transforment aujourd'hui notre monde issu de la "Galaxie Gutenberg ". Nous vivons une époque charnière entre deux âges de l'histoire des communications.

Pourtant, le troisième âge qui s'annonce ne sera pas le monde sans livre que certains se plaisaient naguère à évoquer. De plus en plus, l'imprimé s'impose et poursuit son développement: L'ordinateur s'est jumelé aux imprimantes pour produire une nouvelle génération de livres, de revues et de journaux. Mais le changement invite à une réflexion pour mieux saisir la transformation de notre société à travers celle de sa production imprimée.

\section{Comprendre la place de l'imprimé}

Au Québec, cette réflexion est amorcée déjà depuis plusieurs années. Les travaux de pionniers comme NarcisseE. Dionne, Philéas Gagnon, Aegidius Fauteux ou Antonio Drolet ont tracé, au début de ce siècle, les premiers sentiers vers la bibliographie historique et I'histoire du livre et des bibliothèques. Puis, dans les années 1960 et 1970, dans la mouvance de l'École des Annales, les recherches ont abordé l'étude du livre à travers les métiers, les réseaux de production et de diffusion.

Le circuit qui unit l'écrivain, l'éditeur, l'imprimeur, le libraire et le lecteur est devenu un objet multiforme d'études, un axe de compréhension de la dynamique culturelle de nos sociétés. On a délaissé le récit et l'anecdote pour l'étude des usages de pensée, des pratiques sociales et matérielles reliées au métier des lettres. On s'est arrêté aux stratégies et aux intérêts des groupes associés par des fonctions proches de la communication écrite.
Les travaux publiés au Québec'au cours des années 1970 sur. la librairie 1, les cabinets de lecture ${ }^{2}$, les collèges classiques $^{3}$, la production imprimée ${ }^{4}$ sont venus alimenter cette réflexion. En 1983, un ouvrage collectif publié par Yvan Lamonde ${ }^{5}$ dressait un bilan des recherches de cette décennie. Au cours de la même période, la Bibliothèque nationale du Québec prenait en charge l'inventaire de la production d'imprimés québécois avec la publication des bibliographies courante et rétrospective et celle des travaux de Milada Vlach et de Yolande Buono. On rendait ainsi possible la récupération et la relecture de notre patrimoine imprimé. Les grands projets comme le Dictionnaire biographique du Canada, le Dictionnaire des oeuvres littéraires, ou La Presse québécoise des origines à nos jours ont également apporté des instruments indispensables à la recherche.

Grâce à ces outils, la recherche universitaire s'est développée. On a vu se constituer des groupes d'études explorant diverses dimensions sociales de l'imprimé. Le Centre de recherche en littérature québécoise (CRELIQ) de I'Université Laval et le Centre de recherche en civilisation canadienne-française (CRCCF) de I'Université d'Ottawa ont généré un grand nombre de travaux. A I'Université de Sherbrooke, on a commencé à découvrir l'extraordinaire aventure de I'édition littéraire au Québec, I'Université du Québec, I'Université de Montréal et I'Université Laval, dans leurs départements de littérature, d'histoire, de communications, de sociologie ou de bibliothéconomie, ont jeté les bases d'une féconde réflexion sur la fonction sociale et l'évolution de l'imprimé. Bref, depuis vingt ans au Québec, on assiste, dans plusieurs disciplines, à l'éveil d'un intérêt autour du livre comme objet d'études; ce livre que Lucien Febvre appelait un ferment à l'oeuvre dans notre civilisation.

\section{L'AQÉl : un lieu d'échanges}

L'Association québécoise pour l'étude de l'imprimé (AQÉl) est née en 1987 comme une manifestation de cette vitalité de la recherche sur le livre. L'AQÉl prenait la relève du Groupe de recherche sur l'histoire de l'imprimé (GRHIQ) formé en 1974 par Yvan Lamonde de I'Université McGill. Les rencontres de ce groupe permettaient à des chercheurs universitaires de faire le point, de temps à autre, sur l'état des connaissances dans ce domaine. Mais pendant les années 1980, cellesci débordèrent le cadre de l'histoire pour rejoindre la littérature, la sociologie, la linguistique, la philosophie et les communications.

Des chercheurs de diverses disciplines et de plusieurs institutions, des personnes issues de milieux de l'édition, du journalisme et de la librairie ont voulu créer une association ouverte où I'on pourrait s'informer et multiplier les discussions sur les travaux en cours. Lors de sa fondation à Sherbrooke en avril 1987, l'AQÉl s'est donné quatre objectifs: 1- promouvoir le développement de la recherche sur l'imprimé; 2- diffuser l'information relative aux publications et aux travaux en cours ; 3 - faire des représentations auprès des organismes et des instances reliés à la recherche et à la conservation de l'imprimé; 4- créer des liens de collaboration avec des organisations nationales et internationales qui poursuivent des objectifs similaires.

1. Jean-Louis Roy, Édouard-Raymond Fabre libraire et patriote canadien (1799-1854). Contre l'isolement et la sujétion, Montréal, Hurtubise HMH, 1974, 220p.

2. Marcel Lajeunesse, Les sulpiciens et la vie culturelle à Montréal au XIXe siècle, Montréal, Fides, 1982, 280p.

3. Claude Galarneau, Les collèges classiques au Canada français (1620-1970), Montréal, Fides, 1978, 287p.

4. John Hare et Jean-Pierre Wallot, Les imprimés dans le Bas-Canada 1801-1810, Montréal, Presses de I'Université de Montréal, 1967, XXIII, $383 \mathrm{p}$.

Milada Vlach et Yolande Buono, Laurentiana parus avant 1821, Montréal, Bibliothèque nationale du Québec, 1976, XXVII, 416, 120 p.

Milada Vlach et Yolande Buono, Catalogue collectif des impressions québécoises 17641820, Québec, Publications du Québec, 1984, XXXIII, 251, $195 \mathrm{p}$.

5. Yvan Lamonde, éd. L'imprimé au Québec, aspects historiques (18e-20e siècles), Québec, Institut québécois de recherche sur la culture, 1983, $368 \mathrm{p}$. 
Pour atteindre ces buts, I'AQÉl a lancé un bulletin semestriel d'information dont six numéros sont déjà parus et elle organise des rencontres biannuelles sur les recherches en cours. L'Association a aussi publié un répertoire des chercheurs et une bibliographie des travaux sur l'imprimé qui sera mise à jour annuellement. À l'aútomne de 1991, I'AQÉl organisera un colloque en collaboration avec le ministère des Communications du Québec sur le thème de l' "État québécois et la communication imprimée".

La présence, au sein de l'Association, d'universitaires, de bibliothécaires, de libraires, d'éditeurs et de journalistes constitue la promesse d'échanges fructueux permettant d'éclairer tous les aspects de la production, de la diffusion et de la réception du livre et de l'imprimé en général. Déjà, plusieurs communications présentées aux journées d'échanges de I'AQÉl ont fait l'objet de publications et d'articles dont certains, dans les pages mêmes de Documentation et bibliothèques.

\section{Réfléchir ensemble sur le passé et I'avenir}

L'AQÉl est une association jeune qui n'a pas encore réuni tous ceux et celles qu'elle pourrait intéresser. Elle cherche à rejoindre non seulement les spécialistes dans divers domaines, mais aussi les personnes qui travaillent dans le monde de la documentation, de la production ou de la diffusion documentaire ou qui, simplement, s'intéressent au rôle de l'imprimé dans notre société.

Au-delà des sujets divers et parfois spécialisés qu'elle discute, c'est à une réflexion globale que I'AQÉl invite ses membres. Elle propose en somme une réflexion humaniste sur la culture de l'imprimé et une approche nouvelle du livre et de la dynamique sociale qu'il enrichit et, parfois même, qu'il crée.
L'AQÉl atteindra pleinement ses objectifs si elle parvient à maintenir un faisceau d'intérêts sur ce thème et si elle continue à stimuler une recherche sur l'imprimé. Une recherche qui nous rende plus conscients de nos origines, des défis actuels et qui nous permette de nous tourner résolument vers l'avenir 6 .

Jacques Michon*
président de l'AQÉ

Gilles Gallichan*

vice-président de I'AQÉl

6. Pour obtenir de plus amples renseignements sur l'AQÉl, vous pouvez écrire à:

Association québécoise pour l'étude de l'imprimé, C.P. 92, Sherbrooke (Québec) J1H 5 H5.

* Jacques Michon est professeur de littérature à I'Université de Sherbrooke et Gilles Gallichan est bibliothécaire à l'Assemblée nationale.

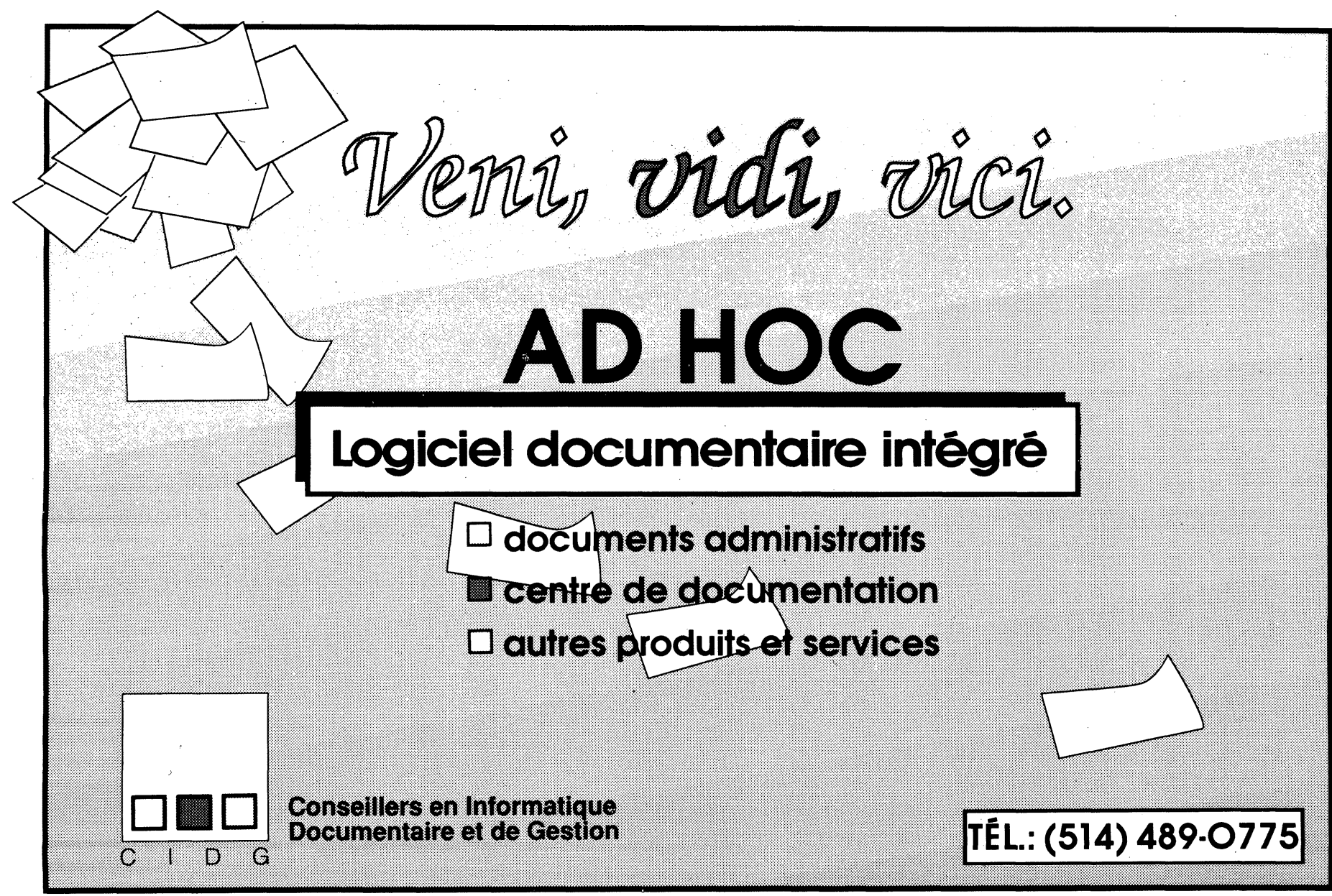

